\title{
Web Structure and Color Arrangement Analysis
}

\author{
Fen $\mathrm{He}^{1, \mathrm{a}}$ \\ ${ }^{1}$ Wuchang Shouyi University, Wuhan 430064,China \\ ahefen@126.com
}

Keywords: Web design; Color arrangement; Internet

\begin{abstract}
With the rapid development of the Internet, as a new discipline Web design is gradually being recognized. Web Design is creative thinking activities of a web designer that artistic planning consciously of objects or constituent elements of the page in accordance with the times, technology and experience that fulfill purpose and requirements of the design. Web design has become an important part of the art of design, an important branch of art and design, which is now rapid development with the development of network technology. Hue, lightness, purity, tone and color and so on constitute the color elements. Web design requirements the area of the page constitution, the formation of the difference between the shape, location, and hue, lightness, purity of a variety of colors to differ from each other by adjusting the color contrast, so as to make color added to the web pages change pages even more colorful, here being evaluated have a unified vision, achieve psychological balance. Web design page layout must properly handle the relationship between structure and color, make sure they are in the packet and integration, symmetry and balance, contrast and unity, emphasizing coordination and prominent, the actual situation and the blank, rhythm and cadence and other complementary aspects.
\end{abstract}

\section{Background of research}

Web design art and design is as a breath of fresh blood, which greatly expanded the range of visual communication design. In just a few years, the web design industry has become an important force in the construction of the entire Internet. [1]Although unlike other traditional design categories such as advertising poster design, packaging designs that has a long history of accumulation, with the development of computer technology, PC, popularity of the network and people's lifestyle changes, this new design category growth rate and prospects are incalculable .

This article explores the shades positioning of web interface design. Researches on web design are quite a lot, but on a separate page devoted to tone issues discussed on the studies to date is still relatively small. With the maturity of broadband network technology, research purely from a technical point cut will inevitably encounter the bottleneck, and the aesthetic nature of the problem starting from web design for the study will be a vast world, and therefore need to be given special attention and discussion. In addition, the study terms of color involves many other disciplines, such as interior design color science, industrial design, color science, color science image design, but study these subjects in terms of color, mostly confined to the general discussion of the concept of big color, instead dedicated to the study of color tone. For the current domestic web colors and color design study the overall situation, most web design theories are put at great length ink enthusiasm in the realization of human-computer interaction technology, network dynamics and other issues, and its essence is a purpose indifferent, while the means to consume enormous energy, the means as an end to the pursuit. Whatever the technology is, it is ultimately serving others. Blind discuss about 
technical often take the site as a web production technology booth, while ignoring the aesthetic subject that is human, their needs. Even if we have a very wide range of web authoring skills, we still found can not catch up with the progress of the rapid development of network technology. Is those dynamic flickering image really necessary? [2]Will the page that will force their way into the viewer's line of sight floating window really help merchandising the role? Maybe this is the issue more worthy of our focus. Although very few journal articles began to pay attention to the tone of the page in question, their basic research sectors still remain in the research stage to do sporadic cases, not yet formed a complete and uniform theoretical system. However, these microscopic studies also not without significance, which anyway laid a foundation for macro-architecture system, and macro research only has the credibility on a microscopic study formation.

\section{Web design introduction}

As the new art of the digital age, web page has the features that being media-related, technical and artistic, as well as a number of features different from traditional art forms. Contemporarily, it is the main carrier of information, it uses digital technology, text, pictures, sound or video transfer the information carried by different styles of page impressions to the viewer. Wherein the color at the center, in a very fine web design allows viewers to patronize many times, we can see the page with color is the key to the success of web design, a well-designed website, it must first resolve color with, so that viewers feel harmonious, decent, and delightful.

\subsection{Web Design Development}

Since 1972, the first International Conference on Computer Communication adopted by the American Vinton Cerf and Robert Kahn developed Transmission Control Protocol and Internet Protocol (TCP / IP), Internet has been in development till today. It has been covering seven continents, in almost all countries of the world Unicom, and has a vast network of nearly hundreds of millions of users. Web art design is a new form of art in the development of Internet and the development of digital technologies. [3]It is the fourth media --Internet as the carrier, network technology and digital technology, in accordance with the design purpose, to follow the laws of art and design, to achieve design objectives and functions, emphasizing the close combination of art and science to create an art and visual communication activity. Web design in the development has gone through three stages: the first stage, as the man-machine communication simple static pages. The second phase, illustrated pages. The third stage, the rapid development of interactive dynamic web pages.

\subsection{The constituent elements of web design}

As we all know, the page is a cause of concern for its information carrier. It is emerging discipline graphic arts and network technology, and usually completes the main elements of visual expression. Web designs elements are carious, which summed up are typography, graphics and graphic design, color design, icons and buttons design, page layout design. This interaction among elements in the page configuration make the rich content of page, the following is brief characteristics of each element.

\section{(1) Text design}

The earliest web design does not include other visual elements, only the text design. Develop till today; the text is still the main carrier for Web page to disseminate information, occupying most of the space in the general layout. Text designs an integral part of the ring in web design, which can not only produce a certain visual stimuli and visual appearance in the mood, but also enable the viewer to produce a rich emotional and psychological mood in reading. Beautiful handwriting giving a beauty, people can read them relaxed and comfortable; new fonts that people can read them 
impressive. These are likely to cause the viewer's interest and improve the effectiveness of the information conveyed.

\section{(2) Graphics and Graphic Design}

Graphics and images have a pivotal position in web design, and now almost web page without graphics and images are quite rare. Web design use graphics and images to bring vitality to the page elements, various forms of graphics and visual images can give people the impression that the page increase the beauty and liveliness, and easy image viewer to understand and accept, is an important means to convey information, such as games, video, scenery spots and some sites need to rely on image to attract visitors, these sites image became a very important factor. If these sites missing image, the whole page will lose vitality. [4]When inserting graphics and images, mainly to see whether it is harmony with other elements, but also pay attention to graphics and images to choose whether to highlight the appropriate web page theme to beautify the same time.

\section{(3) Color design}

Color Design page is involved in both layout design and navigation design of the page, but it also has considerable independence. Moreover, the color page design is first impression left to the viewer, it's comfortable with color or irritability directly impact on the reader's interest in viewing. The different emotional color, the color of strong personality, etc. caused by color sense of form, the performance greatly decide artistic expression, indicating that color with considerable initiative in web design. Visual effects design can produce good color and dominant web pages of a vivid, clear, harmony, and to achieve the effectiveness of visual communication. Color often plays a decisive role in the process of forming the overall visual impression of the page and between pages in. Therefore, mastering the laws of good color pages is a good web design works basic premise.

\section{(4) Icons and buttons design}

Web design icons and buttons are less noticeable but occupy less space, but it is the most important, if the page is missing icons and buttons out of control. Therefore, the design can not be because of the small but ignore its existence, will be a big mistake, it is in web design also highlights the design concept and concentrated. Designers in the design to be style icons and buttons, and hyperlinks to make the icons and buttons appear flashing color dynamic effect on the page, plus a distinctive design, make the page more vivid.

\section{Principle of color and visual}

\subsection{Light and color}

The light and color coexist, only have light can we see color, light and color sense are inseparable. (1) Light and visible light spectrum. Light is an electromagnetic wave in physics. Electromagnetic waves from 0.39 micron to 0.77 micron wavelengths, can cause people to feel that color vision. This range is called the visible spectrum. Said infrared wavelengths longer than 0.7 micron wavelength of less than 0.39 are called ultraviolet.

(2) Light propagation. Just be in the form of fluctuations in a straight line, it has a wavelength and amplitude of the two factors. The length of a different wavelength produces a hue difference. The strength of the size of the different amplitudes produces contrast same hue. There are direct light, reflection, transmission, diffusion, refraction and other forms when spread. Direct light directly into the eye, the visual experience is light color. When the light source illuminate the subject, the light reflected from the surface of the object out of the eye feel the surface color. When irradiated with light, in case of transparent objects like glass, the human eye to see through objects penetrating color. When light propagation, interference by the object, the resulting diffusion, have an impact on the color of the object surface. Such as the generation direction changes through different objects, 
called refraction, reflection of a human eye shade and color of the same object.

\subsection{Object colors}

Natural objects keep changing. Although mostly of these objects, they themselves do not emit light, but have selective absorption, reflection and transmission characteristics of shade. Of course, any object on the shade can not all be absorbed or reflected, therefore, in fact, there is no absolute black or white. Common black, white, gray body color, the white reflectivity of $64 \%-92.3 \%$; gray reflectance is $10 \%-64 \%$; black absorption rate is $90 \%$. On the shade of absorption, reflection or transmission capacity object, popular impact surface texture state, smooth surface, delicate objects, reflecting on the shade stronger, such as mirrors, polished stone surface, silk fabrics. Surface roughness, unevenness, loose objects, easy to produce light diffusion phenomenon, it is the reflection of colored light is weak, such as frosted glass, cloth, sponges. However, the object of absorption and reflectance shade although fixed, while the surface color of the object but it will vary depending on the color of the light source changes, and sometimes even lost its original hue feeling. The so-called object inherent color, in fact is often light that are accustomed to think as.

\subsection{Color mixing}

\section{(1) Addition mixing of light}

It belongs to projection optical mixing. By concentrating the light radiated by a source, we can generate a new shade. For example, when in front of a wall of limestone wall with no light in the dark, eyes can not see it. Wall was red when illuminated only by the red, it is green when only green is illuminated, and red green light at the same time, the wall will seem to be yellow one. The color yellow hue and purity will be in between red and green, the brightness is higher than red, and also higher than green. Since mixing lit the projection become light, so called it is called additive mixing of light.

\section{(2) Subtraction mixing of light}

After the white light went through colored filters, part of the light is reflected and the remaining light is absorbed, reducing portion of the radiation power, the results is the dimming light after being filtering for two times, so it is called subtraction color mixing. In general, dye of strong transparent feature will show significant dimming effect after being mixed. Subtraction mixing the three primary colors is mixed addition of the three primary colors of complementary colors, namely complementary of green red color (magenta), blue-violet complementary color yellow, red complementary color sky blue. Two primary colors are mixed to produce between colors.

\section{Network Interface color design principles}

\subsection{Integrity of color style}

Internet itself has features of being flexible and interactive, where viewers can jump from one page to another page of the site, and therefore, the Internet page is with relative independence. But in terms of the entire site, we should have a design idea that throughout the overall image, in a uniform style outreach to effectively establish a unique image of the site, so as to make the site has integrity. Color, as one of the main forms of visual elements reflects the style of website design; it occupies a heavy load in integrity. The customer or your boss may not discuss the framework for navigation and designers, but they will explore color, because the color is the emotional expression of the public, with good color, can design a harmonious work.

\subsection{Equilibrium of color design}

Balance, from terms of physics, it is symmetrical state between left and right; from the plastic arts of speaking, it is a mutual sense of psychological balance of shape, color, quality and so on in the touching, feeling and visual sense. Network interface color balance is classified in to two forms: 
symmetrical balance and asymmetrical balance. Symmetrical balance is easy to reach equilibrium, characterized by simple and clear picture with the order; it is of calm, stable visual effects, of course, likely to have a dull feeling, lack of vitality. [5]Asymmetrical balance refers to the character color, area color, color location, etc. unequally distributed, make appropriate adjustments according to some space force field to when add color, the feeling is equal in intensity contrast, balanced state. Asymmetrical balance color effects are lively, fresh, very sporty, but much more complex than symmetric equilibrium to grasp the relationship between light and heavy good color, light and dark, advances and retreats, to master. When encounter different page content each color need to do minor adjustments, these adjustments mainly showed in the area of color, color, position, and other elements of coordination and many other issues.

\subsection{Harmony of colors}

Balance of colors derived from the musical term harmony, it refers to the harmonious effect of different colors made in the same space and time. Harmonic is the basic form of color beauty, also the most important form. Color harmony is not intended to make color be the same one, but to coordinate colors of significant differences according to a certain order and unity to stimulate the harmony and beauty of color feel.

\subsection{Emphasis of color contrast}

Color contrast refers to clear color difference when two or more colors arranged together. Difference between the size, shape, location, and hue, purity, brightness and other mental stimulation produce a variety of color. The greater this difference is, the contrast effect is more obvious; while shrinking or reducing this difference, contrast tends to ease. In the web interface design, color contrast can make up for the poor and monotonous color, visual stimulation, distributing color seductive charm that attracts people's attention and interest, to make more design theme distinctive, while the visual effect is more rich.

\section{Summary}

As a branch of graphic design, web design not only contains a lot of elements of graphic design, it also has the features and functionality that traditional graphic design do not have, thus becoming the main means of communication and information dissemination in the 21st Century. Web design itself as a highly comprehensive discipline, involve in much more than just aesthetic design content unilaterally, further comprising content ergonomics, psychology and other aspects.

\section{References}

[1] Cao Jinming, Wangjun. Creative Source - Page Creative, Hubei Fine Arts Publishing House, 2014(6).

[2] Chengchao, Wangjun. Web design and color, Ordnance Industry Press, 2015(3).

[3] Lifang. Examples of Web design and color analysis. Ordnance Industry Press, 2016 (4).

[4] Conglin. Page color composition detail design", Posts \& Telecom Press, 2011(1).

[5] Liqing. See around corners, Web user experience deconstruction, Machinery Industry Press, 2011(5). 